\title{
Light as a Growth Regulator: Controlling Plant Biology with Narrow-bandwidth Solid-state Lighting Systems
}

\author{
Kevin M. Folta ${ }^{1}$ and Kayla Shea Childers \\ Horticultural Sciences Department and the Graduate Program in Plant Molecular and Cellular Biology, \\ University of Florida, 1301 Fifield Hall, Gainesville, FL 32611
}

Additional index words. light, development, photomorphogenesis, static signaling states, LED light

\begin{abstract}
In the mission of plant husbandry, light is a critical yet passive entity. The potential to actively implement dynamic lighting strategies to control plant growth and development holds great promise in the future of plant cultivation. In other words, rather than simply using a single stable light condition to maintain photosynthesis, might it be possible to continually adjust fluence rate, wavelength combinations, and photoperiods to actively manipulate plant morphology and production? Research over the past 100 years suggests that it is so, and today's solid-state, narrow bandwidth lighting platforms offer a unique opportunity to test this hypothesis. The goal of this report is to describe the potential use of light as a growth regulator. Here light-emitting diode technology is well suited for the application, because light quantity, quality, photoperiod, and combinations thereof can be controlled with great precision. Specific light combinations may be adjusted throughout the life of a plant to potentially optimize traits of interest such as synchronization of flowering, maintenance of vegetative growth programs, control of plant stature, or acceleration of juvenility. This report describes the plant photosensory networks and how they sense and respond to light. The connection between light and internal hormone stasis is explored and then extended to questions of designing specific regimes to control plant growth and development. The concept of static signaling states is presented as a means to tightly control plant habits, in essence, using light to stabilize plant signal transduction pathways and their associated outcomes. Finally, the concepts presented are applied to the diploid strawberry Fragaria vesca to demonstrate the usefulness of the approach. These experiments provide proof-of-concept and lay a foundation for further studies.
\end{abstract}

Plant growth and developmental processes are regulated by light quality (color, wavelength), quantity (fluence rate), and photoperiod (duration of illumination). Together these three parameters strongly influence traits of horticultural interest, including plant stature, growth habits, the transition to flowering, and end point plant productivity (reviewed in Casal and Yanovsky, 2005; Chen et al., 2004). Traditionally, plant cultivation activities have been a pawn to prevailing conditions, because little can be done to adjust ambient light conditions in a field setting or greenhouse. Because various artificial light regimes have been designed and adopted the goal has been simply to efficiently provide light energy for photosynthesis, oftentimes placing priority on high fluence rate, lowcost operation, and satisfying the human eye over fine-tuning of conditions to best regulate plant growth. Some of the same fluorescent and incandescent fixtures used in homes and businesses are used to support plant growth in greenhouses and growth chambers. Only a minor subset of luminaries have been designed specifically for plant use.

Although pedestrian lighting strategies have been deployed in greenhouses and growth chambers, research in the laboratory was concurrently characterizing plant photosensory circuits, the associated biochemical transduction networks, and the components relevant to signal integration and response. Here, discrete sensors that interpreted ultraviolet, blue, green, red, and far-red energies were carefully characterized and their con-

Received for publication 24 Mar. 2008. Accepted for publication 13 June 2008.

${ }^{1}$ To whom reprint requests should be addressed; e-mailkfolta@ifas.ufl.edu tributions to plant biology were deciphered in great detail.

However, there existed a palpable uncoupling between the basic science that illustrated these sensory processes in the laboratory plant (Arabidopsis thaliana) and applied plant biology. It was presumed that these sensory pathways were conserved among plants and that translation of arabi-centered paradigms would translate cleanly to other plants. Certainly, shining examples have been presented from other important crop systems such as rice (Zhang et al., 2006), peas (Platten et al., 2005; Weller et al., 2001a), tomato (Weller et al., 2001b), maize (Sheehan et al., 2007), brassicas (Chatterjee et al., 2006), and many other species. Although this translational research is important, few efforts have attempted to extrapolate the findings to the design of plant growth strategies that not only foster plant growth and development, but rather control plant growth and development. In other words, can we begin to apply the lessons learned from basic biology and use light to steer the progress and direction of plant habits to affect desired end products? Lettuce cultivation strives for robust vegetative growth and flowering is undesirable. On the other hand, vegetative growth of strawberry plants is not nearly as desirable as the valued fruits, so it is important to promote flowering to produce the end product. Custom light regimes may be developed to drive plant morphology, development, and productivity from seed to senescence.

Some plant systems are quite amenable to light-driven manipulation. Strawberry is an excellent system to demonstrate how light may be used as a growth regulator in various capacities throughout a plant's life. The ability to set and support large amounts of fruit depends on sufficient vegetative growth, so it is desirable to restrict flowering for a substantial portion of the plant's life while supporting vigorous leaf production. Strawberry plants also runner, so suppressing vegetative growth and channeling the conserved energies into vegetative leaf development in the mother plant could have great benefit both in fruit production and labor savings. Could it be possible to repress or even synchronize flowering in plants so that valuable products come to harvest simultaneously exploiting the best production windows?

\section{THE PLANT PHOTOSENSORY RECEPTORS}

Ever since the advent of agriculture farmers have carefully noted how the environment shaped plant habits and productivity. Once certain cultivated crops left their centers of origin farmers must have witnessed changes in productivity ultimately linked to photoperiodism and seasonality not to the genotypes themselves. Growers were dependent on environmental conditions and here duration of light treatment probably had the most profound role. Other light parameters like light quantity and quality also were relatively static. Today, cultivars are selected based on photoperiod sensitivity for a given region. Still, the ability to skew the ambient spectrum in a field context is difficult. Researchers have used colored mulches to reflect light up from underneath, supplementing additional wavebands that typically would be lost to soil. Changing the reflective mulches to reflect more red and far-red light affects growth of sweet pepper (El-Desouky et al., 2005) and changes the specific accumulation of volatile compounds (Loughrin and Kasperbauer, 2001, 2003). Increased production of aroma compounds in strawberries has been reported based on this simple and passive use of light (Loughrin and Kasperbauer, 2002). 
Over the last century, scientists have observed how specific wavelengths, intensity, and photoperiod together shape plant output. As mentioned earlier, discussions of light effects on plant growth typically conjure reflection on photosynthesis, yet when considering the design of artificial narrow-bandwidth lighting systems, it is important to consider light as a potent regulator of development.

The following paragraphs introduce the plant light sensor set, describing the mechanisms of how particular areas of the spectrum are sensed to shape stature and output. To control plant growth and development with different qualities of light, it is imperative to first contemplate the mechanisms for plant interaction with light environment. By clarifying how light input is sensed, integrated and interpreted, informed guesses at light regimen design can initiate. A short description of the major photosensory systems sets the stage for plant manipulation based on stabilizing flux through these regulatory pathways.

\section{THE PHYTOCHROMES}

Only in the past 50 years has the fundamental regulation of photomorphogenic processes been elucidated. Seminal studies by Garner and Allard noted the role of photoperiodic flowering in Maryland Mammoth tobacco (an excellent historical account is presented in Sage, 1992). Hendricks and Borthwick blazed the trail forward with the identification and eventual isolation of a "Pigment of the Imagination" a red/far-red reversible compound that controlled plant development that would later be designated as phytochrome. The phytochromes are typically thought of as red and far-red reversible photopigments that regulate plant morphology and gene expression (Quail, 2002). However, the phytochrome absorption spectrum also peaks in the blue and extends well into the ultraviolet A portion of the spectrum to these wavebands also stimulate phytochrome-dependent processes. Phytochromes rely on a tetrapyrrole chromophore to absorb photons from the environment (Lagarias and Rapoport, 1980). Biochemical studies indicate that phytochromes are exiled to the cytosol in darkness and then mobilize to the nucleus on illumination (Huq et al., 2003; Kim et al., 2000; Nagy and Schafer, 2000). In one scenario, light activates phytochromes and induces relocalization to the nucleus where they directly participate in modulating gene expression (Martinez-Garcia et al., 2000). Phytochromes exist in the genome as a small multigene family (Clack et al., 1994) containing individual members with expression profiles (Sharrock and Clack, 2002), spectral characteristics (Eichenberg et al., 2000), and dimerization properties (Sharrock and Clack, 2004).

Light activation of phytochromes results in a wide variety of plant responses. Gross morphological alterations include a decrease in stem elongation and an increase in leaf expansion (Parks et al., 2001; Vanvolkenburgh et al., 1990) or coleoptile expansion in monocots. Phytochrome activation initiates plastid development (Horwitz et al., 1988) and controls gene expression in both the choloroplast (Thum et al., 2001) and nucleus (Tepperman et al., 2004). In mature plants, activation of specific phytochromes controls the procession to flowering (Valverde et al., 2004).

One of the best studied roles for phytochromes is their role as a light quality sensor monitoring the balance of red to far-red light in a given context. Leaves are efficient filters of red, blue, and green light. The dominant intracanopy wavelengths are above $700 \mathrm{~nm}$, the far-red region of the spectrum. Plants are extremely sensitive to these wavebands and when the ratio of red to far-red light drops, the plant takes on new growth strategies. Plants in shaded (or artificially far-redenriched) environments show more elongation growth in stems, greater inclination of leaves, and elongation of leaves and petioles (Ballare, 1999; Mullen et al., 2006; Smith and Whitelam, 1997). All of these strategies bring growth behaviors that favor a growing plant's ability to reach above neighbors, allowing it to compete for light resources. Increases in far-red light also can induce flowering in many organisms consistent with its role in Arabidopsis (Valverde et al., 2004).

The phytochromes are exceptional targets for attempts to manipulate plant habits with light. The contrasting effects of red and farred light, the sensitivity to red and far-red balance, and the strong influence on plant morphology, gene expression, and development make the phytochromes, and the red/ far-red part of the spectrum, an important consideration in light regimes designed to control plant behaviors.

\section{THE CRYPTOCHROMES}

The cryptochrome (cry) receptors are blue and ultraviolet A-sensitive receptors that control plant morphology, gene expression, and the transition to flowering. Originally identified genetically by their conspicuously long seedling stem when grown under white or blue conditions [as hy4 (Koornneef et al., 1980)], positional cloning led to the identification of cryl as a blue light photosensor sharing similarities to photolyases (Ahmad and Cashmore, 1993). Like photolyases, cry receptors implement a flavin and pterin chromophore (Lin et al., 1995; Malhotra et al., 1995). However, unlike photolyases, the cry receptors possess no DNA repair activity (Lin et al., 1995; Malhotra et al., 1995), and electrons promoted by light activation function in signal transduction instead (Zeugner et al., 2005). In Arabidopsis signaling, cryptochromes have been identified with discrete roles in early and mature plant physiology (Lin, 2002). Cryptochromes have also been identified in other plants, including tomato (Weller et al., 2001b), pea (Platten et al., 2005), and rice (Zhang et al., 2006). In animal systems, cryptochromes play a central role in regulation of the circadian clock (Panda et al., 2003).
The cryptochromes, like the phyochromes, control many aspects of plant physiology and development. Most studies have investigated cryptochrome's roles during de-etiolation. The cry receptors strongly inhibit stem elongation acting within seconds of illumination and presenting effects that are apparent within $30 \mathrm{sec}$ (Folta and Spalding, 2001). The cryptochrome receptors control gene expression within this early timeframe and contribute to leaf expansion and plastid processes. The most well-described role for the cryptochromes is in flowering, in which blue light activation of the cry 2 receptor is required for timely progression to the reproductive state (Guo et al., 1999; Valverde et al., 2004).

Cryptochrome signaling is likely propagated by several mechanisms. The best described is the active cry receptor's ability to interfere with destruction of factors that positively regulate photomorphogenic progression (Wang et al., 2001). In darkness, the positive regulator HY5 is degraded by ubiquitination and degradation by the $26 \mathrm{~S}$ proteosome (Osterlund et al., 1999). In blue light, cry receptors interact with COP1, a ubiquitin E3 ligase that specifically targets HY5's destruction (Osterlund et al., 1999; Wang et al., 2001). The negative regulation of a negative regulator allows HY5 to initiate the processes associated with blue light such as suppression of stem elongation, expansion of cotyledons, and greening.

Although this is one mechanism, others certainly exist. Cryptochromes function within seconds in association with anion channels at the plasma membrane, so additional layers of nonnuclear function must exist (Folta and Spalding, 2001). The cry receptors also are affected by green light, treatment that may reverse the effects of blue light activation, much like far-red flips phytochrome to an inactive state (Banerjee et al., 2007). This scenario again presents an opportunity to use the light spectrum to specifically affect the activity of the cryptochrome photoreceptor pool, this time using blue and green light in combinations to skew physiological response. Specific effects of green light such as seedling growth promotion and downregulation of plastid transcripts in etiolated seedlings have also been observed that are cryptochrome-independent (reviewed in Folta and Maruhnich, 2007), indicating that these wavebands may also have important roles in controlling plant physiology through a separate sensory system.

\section{PHOTOTROPINS AND THE OTHER LOV-DOMAIN PHOTORECEPTORS}

Without muscles, plants rely on differential growth to adjust the position of organs. The light-inspired plant movements are oftentimes conspicuous such as the leaf movements in Oxalis, heliotropic behaviors in sunflowers, and the simple bending of a stem toward light. A genetic screen for Arabidopsis seedlings deficient in directional growth toward unilateral blue light led to the 
identification of the photosensor responsible for phototropism [nph1, now known as phot1 (Christie et al., 1998; Liscum and Briggs, 1995)]. The phot1 receptor and its counterpart phot 2 regulate a suite of plant processes, ranging from gross morphological changes like leaf expansion (Sakamoto and Briggs, 2002), phototropism (Christie et al., 1998), and early stem elongation (Folta and Spalding, 2001), to cellular processes such as chloroplast position (Kagawa et al., 2001) and stomatal opening (Kinoshita et al., 2001), to regulation of mRNA stability (Folta and Kaufman, 2003). The phot receptors are autophosphorylating serine-threonine kinases that localize to the plasma membrane. The flavin-mononucleotide chromophore is attached to the second of two light-oxygenvoltage (LOV) domains found in the protein (Christie et al., 2002). On absorption of a photon, the receptor undergoes a physical rearrangement that presents the kinase domain for catalysis (Christie et al., 2007; Harper et al., 2003).

In addition to the phototropins, a number of LOV domain proteins have been identified (Cheng et al., 2003), several linked to discrete processes such as the transition to flowering (Imaizumi et al., 2003; Nelson et al., 2000) and maintenance of circadian oscillator period (Somers et al., 2000). These findings suggest that the LOV domain family of proteins regulate highly specialized processes, synchronizing the plant's inner workings to prevailing environmental conditions. The absorption characteristics of this photosensor family have been well studied, and they absorb well in the blue portion of the spectrum with a peak at $450 \mathrm{~nm}$. These studies indicate that blue light provides important environmental information and that adjustment of the blue component of lighting in an artificial environment, even in a minor amount, may be a potent influence in manipulating physiology and production.

\section{PHOTOSYNTHESIS AND CHLOROPLAST FEEDBACK}

Photodevelopment of the chloroplast is mediated by the suite of aforementioned light sensors. However, the chloroplast itself contains chlorophyll (and its precursors), all of which are efficient harvesters of photons. These energized compounds and the processes they drive (photosynthesis, nonphotochemical quenching) affect metabolism and therefore develop metabolic product states that are sensed and used as information within the cell. Retrograde signaling pathways have been well researched in developing seedlings and illustrate the role of chlorophyll precursors in communicating between the chloroplast and nucleus (Nott et al., 2006; Vinti et al., 2000). Similarly, the status of photosynthetic products themselves eventually feedback to the nucleus, modulating gene expression (Baier and Dietz, 2005). In the context of this report, the activity of the chloroplast must be considered. Fortunately or unfortunately, the absorption spectrum for chlorophyll overlaps with those of the aforementioned light sensor families, making it impossible to uncouple their stimulation. The state of the chloroplast becomes more critical under high light conditions, because phy, cry, and phot sensors are likely saturated and the role of photosynthetic metabolism or the problems of excess light energy often become apparent as poor plant performance.

\section{LIGHT ADJUSTS HORMONE LEVELS}

Plant growth regulators are frequently implicated on the response side of photomorphogenic effects. Certainly, a rich history of auxin participation in phototropic curvature has been documented, connecting this hormone to light-mediated responses. Rapid adjustment of gibberellins and auxins from their dark-abundant accumulation to their lower levels in light has been a staple of photomorphogenic hormone research for many decades. Recently, clear roles for adjustment of ethylene, cytokinins, and brassinosteroids have been tightly linked to light responses, serving to link the ambient environment with downstream plant growth.

The most well-studied examples of light remodeling plant response through adjustment of hormone levels have been in several discrete contexts such as early photomorphogenic development and in shade avoidance responses. Light regulation of gibberellin and auxin metabolism (Garcia-Martinez and Gil, 2001; Stavang et al., 2007) and the defects observed in genetic mutants (Peng and Harberd, 1997) reflect the importance of these regulators on stem growth during early development. These same effects were observed in microarray experiments that pointed directly to gibberellins and auxins as modulators of the dark-light transition (Folta et al., 2003; Zhao et al., 2007). Recent studies have resolved the precise mechanism of the integration of light and gibberellin interaction that controls elongation growth (de Lucas et al., 2008; Symons et al., 2008). In mature plants, auxins, gibberellins, and ethylene contribute to the syndrome associated with shade avoidance sensed by the lowering of red/far-red ratio (Morelli and Ruberti, 2000; Pierik et al., 2004; Vandenbussche et al., 2005). Changes in light-induced leaf inclination have been linked to abscisic acid as well (Mullen et al., 2006). In general, when the effect of light as a growth regulator is discussed, it really means the effect of specific light treatments on endogenous hormone stasis than how these changes in growth regulators dovetail with the acute effects of light on plant morphology and gene expression.

\section{PHOTORECEPTOR ACTIVATION GUIDES DEVELOPMENTAL TRANSITIONS}

A tadpole and frog maintain remarkably different body plans, each supporting exploitation of a specific environmental con- dition. The developmental changes that remodel the free swimming legless creature into a fully mobile terrestrial organism are mainly gated by time and metabolism, because animal developmental programs are controlled by internal systems that measure time. The environment plays little role. However, because plants are sessile organisms, the analogous developmental changes that convert a heterotrophic etiolated seedling to a fully functional green plant are regulated by the environment with a lower dependence on time and internal factors. These observations are much more relevant to laboratory model organisms than cultivated perennial plants, because endogenous factors certainly control traits such as juvenility.

The switch from vegetative to reproductive growth habits is an important transition relevant to horticulture in many ways. Ironically, most mechanistic dissection of this process has come from a plant with limited horticultural import, Arabidopsis thaliana (Amasino, 1996; Putterill et al., 2004; Simpson, 2003). Many elegant studies in the last 5 years have described how different wavelengths of light orchestrate the decisions that shape meristematic fate, transforming a vegetative structure into a reproductive one. One of these studies shows that blue light and farred light, acting through cry2 and phyA, respectively, promote flowering in Arabidopsis (Valverde et al., 2004). On the other hand, red light works to antagonize the effects of blue and far-red light. Together, these three independent sensory systems provide a sensitive means to measure the quality and quantity of light, guiding the plant to the most appropriate time to flower.

\section{STATIC SIGNALING STATES}

When considered together, a compelling hypothesis emerges; could the pigments that absorb light in plants and impart biological consequence be activated in a manner that would allow the user to control plant growth with high predictability? Because narrowbandwidth light sources permit tight control of which receptors are being activated, and because photoperiod can be controlled with simple timer (or removed altogether), it might be possible to steer internal stasis by establishing static signaling states. In such cases, as many environmental variables would be removed as possible, making light quality and quantity the only fluctuating factors. A given set of treatments would establish a relationship with the plant's genetic and developmental context, generating a metabolic and developmental pattern that could be maintained until acted on by a change in light conditions. It is possible that once a plant is trained to a set of static environmental variables, the shift to other conditions would induce acute responses such as tight synchronization of flowering or fruit ripening. Such hypotheses could not be tested in a shortlived species like Arabidopsis, because developmental change is constant. However, an herbaceous perennial like strawberry 
would make an excellent subject for study. This report provides a foundation of these concepts by simply monitoring the baseline of strawberry developmental habits in monochromatic light conditions.

\section{PROOF OF CONCEPT IN DIPLOID STRAWBERRY (Fragaria vesca)}

The implementation of light as a growth regulator can be tested in the laboratory with Fragaria vesca, the diploid woodland strawberry. This system is well suited to this study based on analysis of the literature and many attributes of the plant's fundamental biology (Folta and Davis, 2007; Folta and Dhingra, 2006; Oosumi et al., 2006). Observations of photoperiod and strawberry flowering date back to Duschene in the 17th century (Darrow, 1966). Contemporary studies of photoperiodic flowering in strawberry were conducted by Darrow and Waldo concurrent with the groundbreaking work in tobacco. Throughout the 20th century, bench scientists to breeders noted the effects of light in the regulation of flowering in cultivated strawberry, because the octoploid background presented a formidable yet economically pivotal background to dissect this response.

Strawberry is an excellent system to study the effects of light on a perennial plant because of the distinct fates of meristematic tissues. Environmental and genetic cues influence the emergence of leaves, inflorescences, or rapidly elongating stolons. Perhaps, most importantly, strawberry is a member of the Rosaceae family, a family containing high-value tree fruit crops and ornamentals. Although their large stature, long juvenility, and dormancy requirements limit their use as a laboratory experimental system, strawberry's compact growth habits and rapid cycling permit its study within the confines of an light-emitting diode (LED)based chamber.

Several studies have examined the effect of narrow-bandwidth light on strawberry physiology. Vince-Prue and colleagues noted that petiole elongation and flowering could be affected by adjusting phytochrome response (Vince-Prue and Guttridge, 1973). A report by Yanagi and colleagues illustrated how light integrates with flowering in strawberry using the CHI-24-1 genotype. This line flowers under 24-h light, when noninductive long days (16-h light, 8-h dark) are supplemented with an incandescent daylength extension (Yanagi et al., 2006). A careful dissection of the wavelengths driving this response was conducted using colored florescent and LED light. These tests concluded that the far-red component of light $(735 \mathrm{~nm})$ was driving floral initiation, likely acting through phytochrome. Blue and red had no effect. If related to the Arabidopsis model, far-red addition would limit phyB action and enhance phyA activity, leading to flowering. In this sense, the results are consistent with what happens in the model, yet the interpretation is clouded by the fact that these experiments were performed without photoperiod. However, the clear use of nar- row-bandwidth light to steer development is clearly illustrated.

In this report, the effect of narrow-bandwidth light on plant stature, runnering, and flowering is presented. Here, the genotype used in Hawaii-4 (FRA197; PI 551572). This genotype is important because its ease of transformation (Haymes and Davis, 1998; Oosumi et al., 2006) makes it ideal for in planta studies of gene effects. Moreover, an advanced inbred line from this genotype is the subject for diploid strawberry genome sequencing efforts (J. Slovin, personal communication). It is likely that this particular line will be the subject of a multitude of analyses going forward, so it is important to establish a rapid-cycling protocol as well as define a physiological baseline for later genetic studies or evaluation of transgene effects. The Hawaii-4 genotype has been described as a day-neutral plant, producing flowers under all photoperiods (Oosumi et al., 2006).

A basic experiment was established in which soilborne flats of plants were placed into narrow-bandwidth light conditions. The plant materials were obtained as runners from greenhouse-grown plants at the two-leaf stage. These propagules were cut from the mother plant and firmly pressed into moist planting medium (Pro-Mix BX; Premier Horticulture; Quakertown, PA) under plastic cover for 1 week under ambient fluorescent light conditions. After 1 week, the leaves were excised by cutting them away from the crown at the base of the petiole as described (Vince-Prue et al., 1976). Random crowns were moved to one of four conditions with a 16-h daylength and a total photon fluence rate of $100 \mu \mathrm{mol} \cdot \mathrm{m}^{-2} \cdot \mathrm{s}^{-1}$, shown in Figure 1. This photon fluence rate was distributed differently in the four conditions, the first being $100 \%$ blue, the second $66 \%$ blue, $34 \%$ red, the third, $34 \%$ blue, $66 \%$ red, and the fourth, $100 \%$ red. The light source was $20 \mathrm{~cm}$ from the soil. The wavelength and bandwidth of the light sources are presented in Figure 2. Illumination was provided by $3-\mathrm{W}$ LED devices (American Bright LED, Chino, CA) driven by a custom pulse wave-modulated circuit for stable fluence rate control and thermally managed by actively cooled heat sinks identical to those in Folta et al. (2005). The growth chambers used were each $27 \mathrm{~cm}^{3}$ constructed from HDF-melamine and are lined with mirror to ensure complete light scattering. Plants were maintained with the addition of municipal water every 3 to $4 \mathrm{~d}$ supplemented with $1 / 10 \times$ Peter's fertilizer. After $40 \mathrm{~d}$ in experimental conditions, the plants were weighed and dissected, and their individual parts were digitally imaged on a flat-bed scanner at high resolution. Measurement of growth (elongation, area) was assessed using the open source Image Tool 3.0 (http://ddsdx.uthscsa. edu/dig/itdesc.html) against simultaneously scanned size standards.

A series of horticulturally relevant metrics were assessed for each of the growth conditions. In general, all plants were vigorous and healthy with the exception of perhaps one or two per flat that did not initiate leaf emergence from the crown until late in the experiment. These outliers brought substantial variability to the measurements; yet, they were consistent between treatments. The variables assessed were total fresh weight, petiole length, petiole fresh weight, leaflet number, leaflet area, root length, number of runners, number of flowers, root fresh weight, and aerial fresh weight. A subset of these results are presented in Figure 3.

Although the fluence rate is constant between light conditions, some effects of light quality are observed. Moreover, the most obvious effects come from synergistic stimulation of multiple light sensory pathways, because significant changes in mass, elongation growth, and development are observed when blue and red light are administered in combination. To the eye, the blue light-grown plants were compact and runnerless, whereas the red light-grown plants were highly tall, runnering, and flowering. Intermediate conditions were comparable, generally featuring more leaf expansion and compact growth habits, whereas both flowering and runnering. Specifically, fresh weight and runnering in combination light treatments were similar yet significantly greater than either monochromatic light condition. Blue light strongly suppresses elongation growth, because the end point length of petioles is inversely proportional to the amount of blue light administered. Although not measured, the leaves in blue light conditions clearly thicker. Plants grown under red light maintained long thin petioles and lower levels of leaf expansion. It was observed that the runners in constant red light were much thinner than those observed in other conditions. The major effects were seen as differences in petiole length and fresh weight, whereas the number of petioles per plant remained the same. Figure $3 \mathrm{~A}-\mathrm{C}$ present metrics of petiole abundance and size, factors associated with general plant architecture. The results reflect the strong petiole elongation suppression by blue light, resulting in a greater mass and length per petiole in conditions in which blue light is lost with increasing red. The total number of petioles did not vary significantly between treatments.

In general, the plants in mixed red-blue conditions obtained the greatest total fresh weight (Fig. 3D) and root mass (not shown). The differences observed are even greater when the excessive petiole elongation growth and mass under constant red (Figs. $3 \mathrm{~A}$ and $3 \mathrm{C}$ ) are taken into consideration. The greater plant fresh weight is likely associated with the greater leaf area achieved under these conditions (Fig. 3E). Although $34 \%$ blue, $66 \%$ red conditions had a comparable number of petioles and leaflets, the red led to a significantly higher mass in leaves (not shown). Although not shown here, the plants in red light supplemented with blue gained the most vegetative growth over the shortest time and were the first to flower. These conditions should be considered as the 
starting point for further investigations and even expanded to cultivated varieties.

In terms of applications to research, growth in constant strong blue light offers many advantages. The plants maintain a smaller stature and do not runner. When maintaining a large population of experimental germplasm, these attributes are especially favorable, because it allows more plants per unit of space and minimizes the labor required to remove runners. It may also be possible to maintain vegetative plants in blue conditions and then induce flowering by adding red light. Such practices may be extremely useful in synchronizing flowering or fruit set.

\section{NEXT STEPS, POTENTIAL APPLICATIONS, AND CONCLUSIONS}

The best way to test if light establishment of static signaling states could be used as a growth regulator would be to study LED light effects while minimizing other environmen-

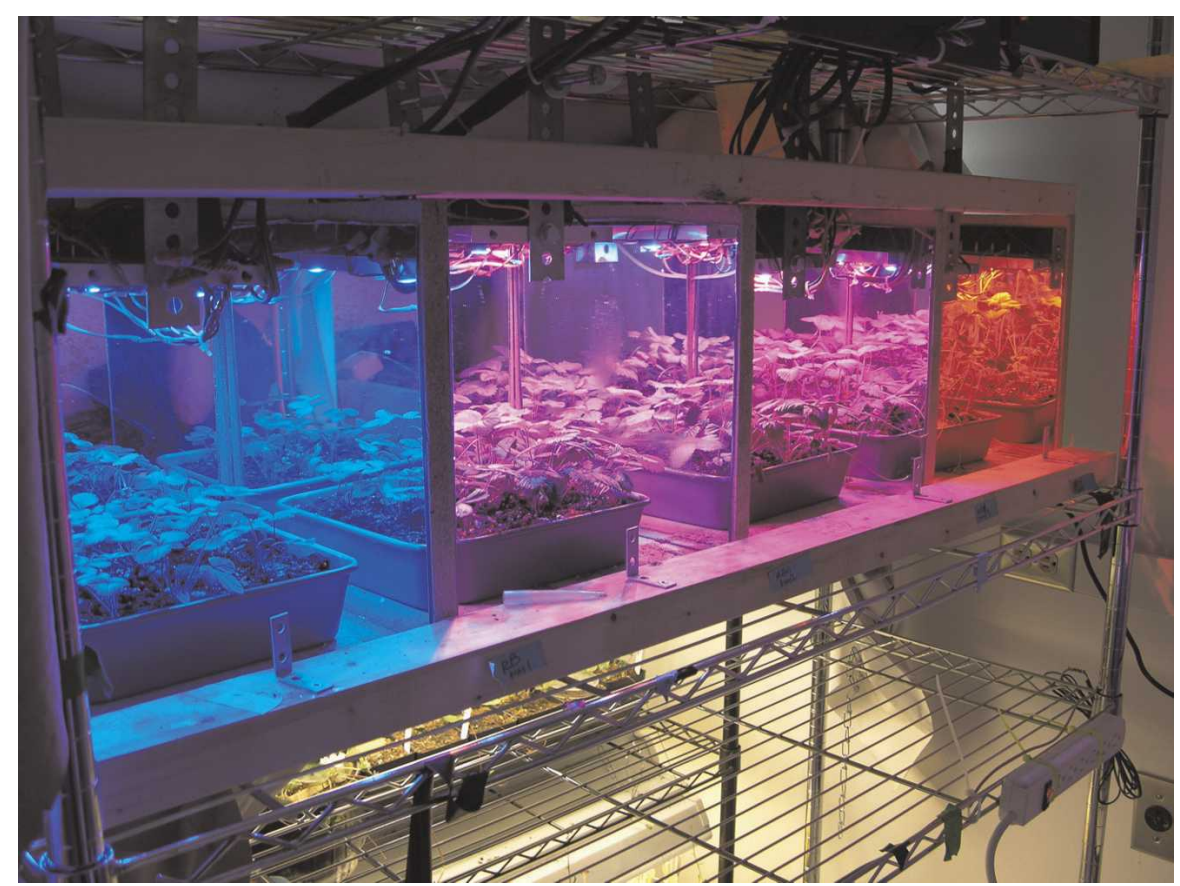

Fig. 1. Diploid strawberry plants were grown under four different spectral conditions and measured for traits of horticultural relevance. The fluence rate was kept constant at $100 \mu \mathrm{mol} \cdot \mathrm{m}^{-2} \cdot \mathrm{s}^{-1}$ and split between blue and red, resulting in $100 \%$ blue, $66 \%$ blue $34 \%$ red, $34 \%$ blue, $66 \%$ red, and $100 \%$ red light treatments. tal cues. The experiments described here were performed with a $16 / 8$ light/dark photoperiod. Future work will be performed under constant illumination to abrogate the potent effects of the photoperiod pathway. Additional mechanisms will ensure uniform and consistent watering as well as humidity control. With plant systems in a constant state, the effects of a shift in the light environment may be most readily observed and accurately delivered.

The future of using light to manipulate plant habits and productivity is filled with opportunities. Cultivated strawberry breeding relies on careful consideration of the pedigrees of elite cultivars and productive genotypes in breeding populations. Although traits like fruit size and uniformity may make two parental lines excellent choices, they may have photoperiodic limitations to their ability to be crossed, at least on demand. Here, LED-based lighting regimes may be implemented to override photoperiodic ten- dencies, inducing synchronous flowering and speeding breeding. Also relevant to strawberry cultivation, most production plants are installed as young daughter plants every year, relying on robust vegetative propagation from nurseries specializing in strawberry plant production. Here, day-neutral and everbearing varieties may be prone to flowering in these settings, again causing expenditure on labor and skewing production away from runners and toward nonmarketable fruit. Could the application of specific wavelengths be used to suppress the flowering response and encourage runnering, purely as a supplement to ambient illumination? Current research suggests that it is likely, and these exciting concepts are poised to be extended to other crop systems. It is imaginable that highvalue specialty crops with photoperiod sensitivity, like herbs, could be manipulated to adopt an empirically designed growth habit driven by a prescription of specific light conditions.

Another application of these technologies lies in supporting human exploration. Travel and colonization of space and remote areas of our own planet will require humans to grow their own food. Most importantly, a balanced diet will depend on the capacity to control production of vegetative and reproductive plant products. A substantial body of work has examined the effects of LEDs on plant growth and development, specifically with respect to growth in space-borne environments (Brown et al., 1995; Goins et al., 1997; Yorio et al., 2001). For instance, it will be important to keep lettuce-producing leaves and strawberries-producing fruits, yet at times, it will be necessary to induce flowering in lettuce to produce seeds for subsequent generations. In these scenarios, artificial lighting programs with known effects on plant physiology and productivity will be essential.

The cost per lumen of solid-state lighting has decreased dramatically over the last decade. The sheer volume of efficient LEDlighting modules being produced along with improvements in emitter design has brought the ability generate more light, more efficiently, and at a lower cost. As breakthroughs in the molecular-genetic control of plant physiology and development continue, a complementary effort in design of speciesspecific lighting regimes may help maximize

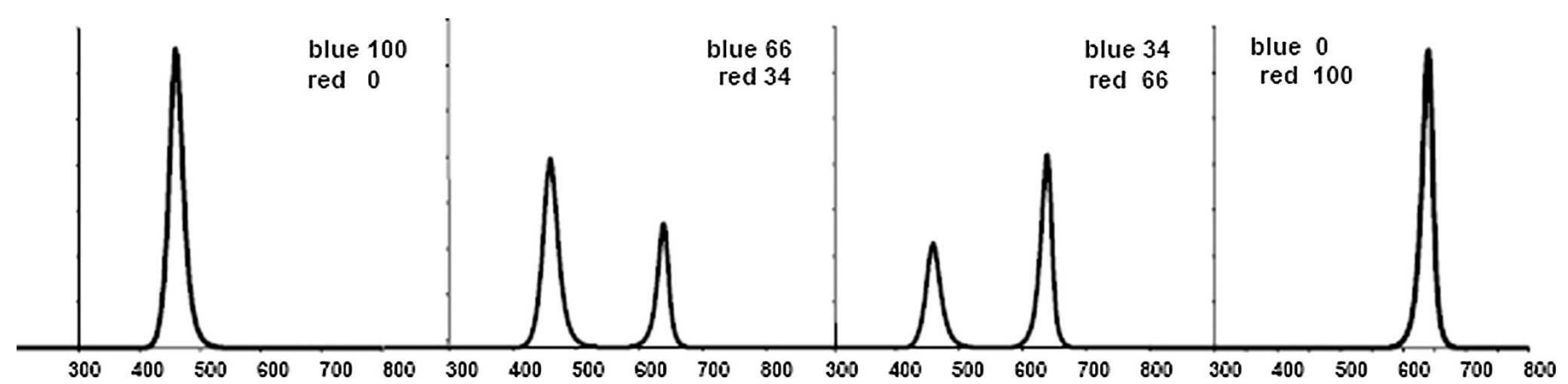

Fig. 2. The spectral conditions of the light experiment. The y-axis is the relative photon fluence rate per nanometer of wavelength. 

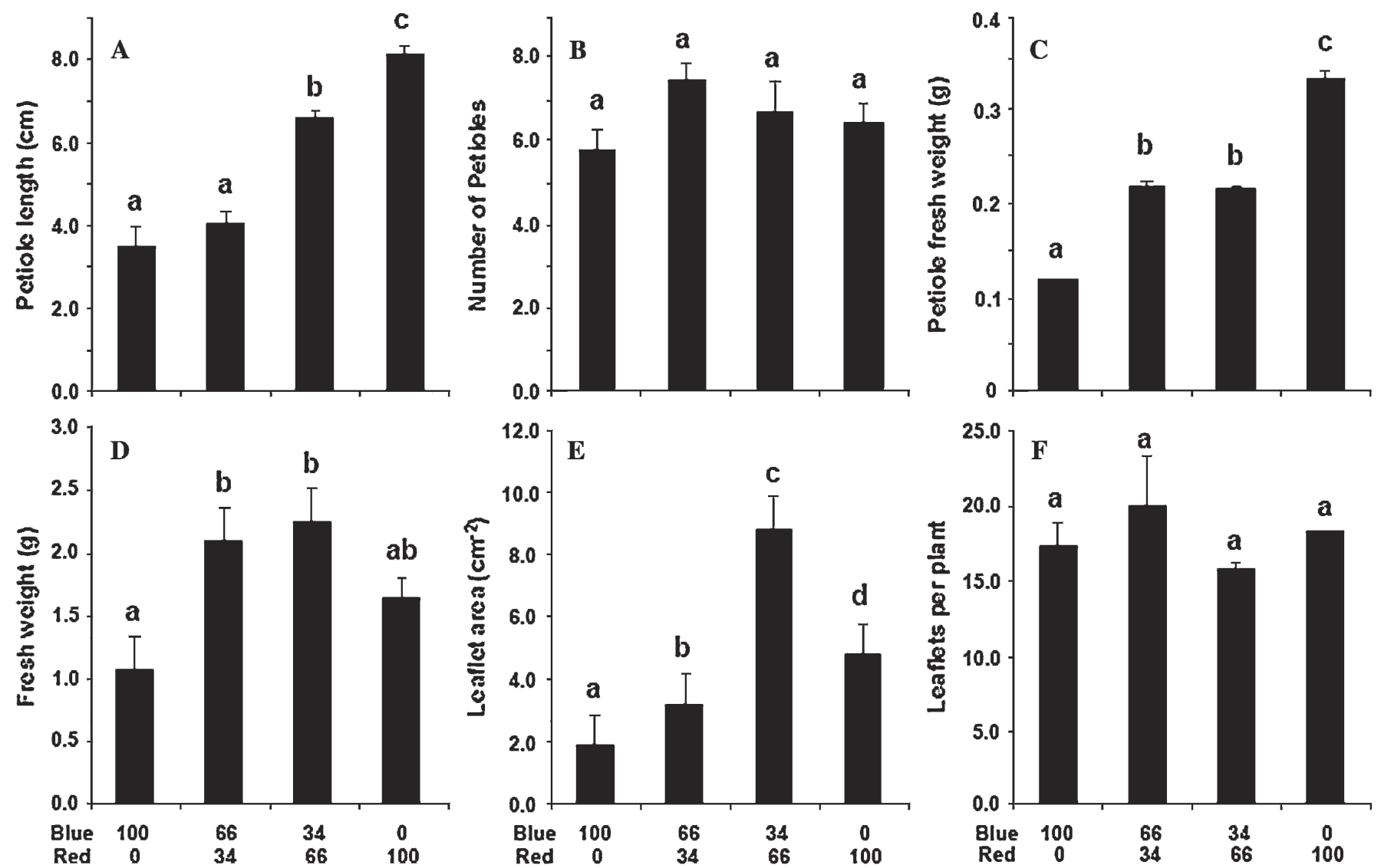

Fig. 3. The effect of blue/red light mixture on plant growth and development. After $40 \mathrm{~d}$ in the specific treatment, the plants were weighed and dissected. Measurements were taken and conditions compared. The panels depict the growth differences observed in (A) petiole length (n greater than 58); (B) number of petioles per plant (n greater than 9); (C) petiole fresh weight (n greater than 58); (D) total fresh weight of the plant (n greater than 9); (E) the mean leaflet area (n greater than 138); and (F) number of leaflets per plant (n greater than 9). Error bars represent standard error of the mean, and statistically significant $(P<$ 0.05 ) differences are noted by different letters (analysis of variance and a posteriori Tukey's test).

our control of plant productivity, increasing food quality and consistency while limiting labor costs and environmental impacts of cultivation.

\section{Literature Cited}

Ahmad, M. and A.R. Cashmore. 1993. HY4 gene of $A$. thaliana encodes a protein with characteristics of a blue-light photoreceptor. Nature 366:162-166.

Amasino, R.M. 1996. Control of flowering time in plants. Curr. Opin. Genet. Dev. 6:480-487.

Baier, M. and K.J. Dietz. 2005. Chloroplasts as source and target of cellular redox regulation: A discussion on chloroplast redox signals in the context of plant physiology. J. Expt. Bot. 56:1449-1462.

Ballare, C.L. 1999. Keeping up with the neighbours: Phytochrome sensing and other signalling mechanisms. Trends Plant Sci. 4:97-102.

Banerjee, R., E. Schleicher, S. Meier, R. Munoz Viana, R. Pokorny, M. Ahmad, R. Bittl, and A. Batschauer. 2007. The signaling state of Arabidopsis cryptochrome 2 contains flavin semiquinone. J. Biol. Chem. 282:14916-14922.

Brown, C.S., A.C. Schuerger, and J.C. Sager. 1995. Growth and photomorphogenesis of pepper plants under red light-emitting-diodes with supplemental blue or far-red lighting. J. Amer. Soc. Hort. Sci. 120:808-813.

Casal, J.J. and M.J. Yanovsky. 2005. Regulation of gene expression by light. Int. J. Dev. Biol. 49:501-511.
Chatterjee, M., P. Sharma, and J.P. Khurana. 2006. Cryptochrome 1 from Brassica napus is upregulated by blue light and controls hypocotyl/ stem growth and anthocyanin accumulation. Plant Physiol. 141:61-74.

Chen, M., J. Chory, and C. Fankhauser. 2004 Light signal transduction in higher plants. Annu. Rev. Genet. 38:87-117.

Cheng, P., Q. He, Y. Yang, L. Wang, and Y. Liu. 2003. Functional conservation of light, oxygen, or voltage domains in light sensing. Proc. Natl. Acad. Sci. USA 100:5938-5943.

Christie, J.M., S.B. Corchnoy, T.E. Swartz, M. Hokenson, I.S. Han, W.R. Briggs, and R.A. Bogomolni. 2007. Steric interactions stabilize the signaling state of the LOV2 domain of phototropin 1. Biochemistry 46:9310-9319.

Christie, J.M., P. Reymond, G.K. Powell, P. Bernasconi, A.A. Raibekas, E. Liscum, and W.R. Briggs. 1998. Arabidopsis NPH1: A flavoprotein with the properties of a photoreceptor for phototropism. Science 282:1698-1701.

Christie, J.M., T.E. Swartz, R.A. Bogomolni, and W.R. Briggs. 2002. Phototropin LOV domains exhibit distinct roles in regulating photoreceptor function. Plant J. 32:205-219.

Clack, T., S. Mathews, and R.A. Sharrock. 1994. The phytochrome apoprotein family in Arabidopsis is encoded by five genes: The sequences and expression of PHYD and PHYE. Plant Mol. Biol. 25:413-427.

Darrow, G. 1966. The strawberry: History breeding and physiology. Holt, Rinehart and Winston, New York, NY. de Lucas, M., J.M. Daviere, M. Rodriguez-Falcon, M. Pontin, J.M. Iglesias-Pedraz, S. Lorrain, C. Fankhauser, M.A. Blazquez, E. Titarenko, and S. Prat. 2008. A molecular framework for light and gibberellin control of cell elongation. Nature 451:480-484.

Eichenberg, K., I. Baurle, N. Paulo, R.A. Sharrock, W. Rudiger, and E. Schafer. 2000. Arabidopsis phytochromes $\mathrm{C}$ and $\mathrm{E}$ have different spectral characteristics from those of phytochromes A and B. FEBS Lett. 470:107-112.

El-Desouky, S.A., H.M. Abd El-Dayem, A.L. Wanas, and M.A. Mady. 2005. Studies on photomorphogenesis in some economical plants. I-Vegetative growth of tomato and sweet pepper plants as affected by red and far-red light reflected from colored plastic mulches. Annals of Agricultural Science, Moshtohor 43. Faculty of Agriculture, University of Ain Shams, Cairo, Egypt.

Folta, K.M. and T.M. Davis. 2007. Transformation systems to study gene function. In: Takeda, F. (ed.). Fragaria. 6th North American Strawberry Symposium 2007 Berry Conference.

Folta, K.M. and A. Dhingra. 2006. Transformation of strawberry: The basis for translational genomics in Rosaceae. In Vitro Cellular and Developmental Biology-Plant 42:482-490.

Folta, K.M. and L.S. Kaufman. 2003. Phototropin 1 is required for high-fluence blue-light-mediated mRNA destabilization. Plant Mol. Biol. 51:609-618.

Folta, K.M., L. Koss, R. McMorrow, H.-H. Kim, J.D. Kenitz, R. Wheeler, and J.C. Sager. 2005. 
Design and fabrication of LED-based light arrays for plant research. BMC-Plant Biol. 5:17-28.

Folta, K.M., and S.A. Maruhnich. 2007. Green light: A signal to slow down or stop. J. Exp. Bot. 58:3099-3111.

Folta, K.M., M.A. Pontin, G. Karlin-Neumann, R. Bottini, and E.P. Spalding. 2003. Genomic and physiological studies demonstrate roles for auxin and gibberellin in the early phase of cryptochrome 1 action in blue light. Plant J. 36:203-214

Folta, K.M. and E.P. Spalding. 2001. Unexpected roles for cryptochrome 2 and phototropin revealed by high-resolution analysis of blue light-mediated hypocotyl growth inhibition. Plant J. 26:471-478.

Garcia-Martinez, J.L. and J. Gil. 2001. Light regulation of gibberellin biosynthesis and mode of action. J. Plant Growth Regul. 20:354-368.

Goins, G., N. Yorio, M. Sanwo, and C. Brown. 1997. Photomorphogenesis, photosynthesis, and seed yield of wheat plants grown under red light-emitting diodes (LEDs) with and without supplemental blue lighting. Experimental Botany 48:1407-1413.

Guo, H., H. Duong, N. Ma, and C. Lin. 1999. The Arabidopsis blue light receptor cryptochrome 2 is a nuclear protein regulated by a blue lightdependent post-transcriptional mechanism. Plant J. 19:279-287.

Harper, S.M., L.C. Neil, and K.H. Gardner. 2003. Structural basis of a phototropin light switch. Science 301:1541-1544.

Haymes, K.M. and T.M. Davis. 1998. Agrobacterium mediated transformation of 'Alpine' Fragaria vesca, and transmission of transgenes to R1 progeny. Plant Cell Rep. 17:279-283.

Horwitz, B.A., W.F. Thompson, and W.R. Briggs. 1988. Phytochrome regulation of greening in Pisum-chlorophyll accumulation and abundance of messenger-RNA for the light-harvesting chlorophyll $\mathrm{A} / \mathrm{B}$ binding proteins. Plant Physiol. 86:299-305.

Huq, E., B. Al-Sady, and P.H. Quail. 2003. Nuclear translocation of the photoreceptor phytochrome B is necessary for its biological function in seedling photomorphogenesis. Plant J. 35:660-664.

Imaizumi, T., H.G. Tran, T.E. Swartz, W.R. Briggs, and S.A. Kay. 2003. FKF1 is essential for photoperiodic-specific light signalling in Arabidopsis. Nature 426:302-306.

Kagawa, T., T. Sakai, N. Suetsugu, K. Oikawa, S. Ishiguro, T. Kato, S. Tabata, K. Okada, and M. Wada. 2001. Arabidopsis NPL1: A phototropin homolog controlling the chloroplast high-light avoidance response. Science 291:2138-2141.

Kim, L., S. Kircher, R. Toth, E. Adam, E. Schafer, and F. Nagy. 2000. Light-induced nuclear import of phytochrome-A:GFP fusion proteins is differentially regulated in transgenic tobacco and Arabidopsis. Plant J. 22:125-133.

Kinoshita, T., M. Doi, N. Suetsugu, T. Kagawa, M. Wada, and K. Shimazaki. 2001. Phot1 and phot 2 mediate blue light regulation of stomatal opening. Nature 414:656-660.

Koornneef, M., E. Rolff, and C. Spruit. 1980. Genetic control of light-inhibited hypocotyl elongation in Arabidopsis thaliana (L.). Heynh. Z. Pflanzenphysiol. 100:147-160.

Lagarias, J.C. and H. Rapoport. 1980. Chromopeptides from phytochrome-The structure and linkage of the Pr form of the phytochrome chromophore. J. Amer. Chem. Soc. 102:48214828.

Lin, C. 2002. Blue light receptors and signal transduction. Plant Cell 14(Suppl):S207-S225.
Lin, C., D.E. Robertson, M. Ahmad, A.A. Raibekas, M.S. Jorns, P.L. Dutton, and A.R. Cashmore. 1995. Association of flavin adenine dinucleotide with the Arabidopsis blue light receptor CRY1. Science 269:968-970.

Liscum, E. and W.R. Briggs. 1995. Mutations in the NPH1 locus of Arabidopsis disrupt the perception of phototropic stimuli. Plant Cell 7:473-485.

Loughrin, J.H. and M.J. Kasperbauer. 2001. Light reflected from colored mulches affects aroma and phenol content of sweet basil (Ocimum basilicum L.) leaves. J. Agr. Food Chem. 49: 1331-1335.

Loughrin, J.H. 2002. Aroma of fresh strawberries is enhanced by ripening over red versus black mulch. J. Agr. Food Chem. 50:161165.

Loughrin, J.H. 2003. Aroma content of fresh basil (Ocimum basilicum L.) leaves is affected by light reflected from colored mulches. J. Agr. Food Chem. 51:2272-2276.

Malhotra, K., S.T. Kim, A. Batschauer, L. Dawut, and A. Sancar. 1995. Putative blue-light photoreceptors from Arabidopsis thaliana and Sinapis alba with a high degree of sequence homology to DNA photolyase contain the two photolyase cofactors but lack DNA repair activity. Biochemistry 34:6892-6899.

Martinez-Garcia, J.F., E. Huq, and P.H. Quail. 2000. Direct targeting of light signals to a promoter element-bound transcription factor. Science 288:859-863.

Morelli, G. and I. Ruberti. 2000. Shade avoidance responses. Driving auxin along lateral routes. Plant Physiol. 122:621-626.

Mullen, J.L., C. Weinig, and R.P. Hangarter. 2006. Shade avoidance and the regulation of leaf inclination in Arabidopsis. Plant Cell Environ. 29:1099-1106.

Nagy, F. and E. Schafer. 2000. Nuclear and cytosolic events of light-induced, phytochrome-regulated signaling in higher plants. EMBO J. 19:157-163.

Nelson, D.C., J. Lasswell, L.E. Rogg, M.A. Cohen, and B. Bartel. 2000. FKF1, a clock-controlled gene that regulates the transition to flowering in Arabidopsis. Cell 101:331-340.

Nott, A., H.S. Jung, S. Koussevitzky, and J. Chory. 2006. Plastid-to-nucleus retrograde signaling. Annu. Rev. Plant Biol. 57:739-759.

Oosumi, T., H.A. Gruszewski, L.A. Blischak, A.J. Baxter, P.A. Wadl, J.L. Shuman, R.E. Veilleux, and V. Shulaev. 2006. High-efficiency transformation of the diploid strawberry (Fragaria vesca) for functional genomics. Planta 223:1219-1230.

Osterlund, M.T., L.H. Ang, and X.W. Deng. 1999. The role of COP1 in repression of Arabidopsis photomorphogenic development. Trends Cell Biol. 9:113-118.

Panda, S., J.B. Hogenesch, and S.A. Kay. 2003. Circadian light input in plants, flies and mammals. Novartis Found Symp 253:73-82; discussion 82-88, 102-109, 281-284.

Parks, B.M., K.M. Folta, and E.P. Spalding. 2001. Photocontrol of stem growth. Curr. Opin. Plant Biol. 4:436-440.

Peng, J. and N.P. Harberd. 1997. Gibberellin deficiency and response mutations suppress the stem elongation phenotype of phytochrome-deficient mutants of Arabidopsis. Plant Physiol. 113:1051-1058.

Pierik, R., M.L. Cuppens, L.A. Voesenek, and E.J. Visser. 2004. Interactions between ethylene and gibberellins in phytochromemediated shade avoidance responses in tobacco. Plant Physiol. 136:2928-2936.
Platten, J.D., E. Foo, F. Foucher, V. Hecht, J.B. Reid, and J.L. Weller. 2005. The cryptochrome gene family in pea includes two differentially expressed CRY2 genes. Plant Mol. Biol. 59:683-696.

Putterill, J., R. Laurie, and R. Macknight. 2004. It's time to flower: The genetic control of flowering time. Bioessays 26:363-373.

Quail, P.H. 2002. Photosensory perception and signalling in plant cells: New paradigms? Curr. Opin. Cell Biol. 14:180-188.

Sage, L.C. 1992. Pigment of the imagination. Academic Press, San Diego, CA.

Sakamoto, K. and W.R. Briggs. 2002. Cellular and subcellular localization of phototropin 1. Plant Cell 14:1723-1735.

Sharrock, R.A. and T. Clack. 2002. Patterns of expression and normalized levels of the five Arabidopsis phytochromes. Plant Physiol. 130: 442-456.

Sharrock, R.A. 2004. Heterodimerization of type II phytochromes in Arabidopsis. Proc. Natl. Acad. Sci. USA 101:11500-11505.

Sheehan, M.J., L.M. Kennedy, D.E. Costich, and T.P. Brutnell. 2007. Subfunctionalization of PhyB1 and PhyB2 in the control of seedling and mature plant traits in maize. Plant J. 49:338-353.

Simpson, G.G. 2003. Evolution of flowering in response to day length: Flipping the CONSTANS switch. Bioessays 25:829-832.

Smith, H. and G.C. Whitelam. 1997. The shade avoidance syndrome: Multiple responses mediated by multiple phytochromes. Plant Cell Environ. 20:840-844

Somers, D.E., T.F. Schultz, M. Milnamow, and S.A. Kay. 2000. ZEITLUPE encodes a novel clock-associated PAS protein from Arabidopsis. Cell 101:319-329.

Stavang, J.A., O. Junttila, R. Moe, and J.E. Olsen. 2007. Differential temperature regulation of GA metabolism in light and darkness in pea. J. Expt. Bot. 58:3061-3069.

Symons, G.M., J.J. Smith, T. Nomura, J.E. Olsen, N.W. Davies, T. Yokota, and J.B. Reid. 2008. The hormonal regulation of de-etiolation. Planta 227:1115-1125.

Tepperman, J.M., M.E. Hudson, R. Khanna, T. Zhu, S.H. Chang, X. Wang, and P.H. Quail. 2004. Expression profiling of phyB mutant demonstrates substantial contribution of other phytochromes to red-light-regulated gene expression during seedling de-etiolation. Plant J. 38:725-739.

Thum, K.E., M. Kim, D.A. Christopher, and J.E. Mullet. 2001. Cryptochrome 1, cryptochrome 2 , and phytochrome a co-activate the chloroplast psbD blue light-responsive promoter. Plant Cell 13:2747-2760.

Valverde, F., A. Mouradov, W. Soppe, D. Ravenscroft, A. Samach, and G. Coupland. 2004. Photoreceptor regulation of CONSTANS protein in photoperiodic flowering. Science 303:1003-1006.

Vandenbussche, F., R. Pierik, F.F. Millenaar, L.A. Voesenek, and D. Van Der Straeten. 2005. Reaching out of the shade. Curr. Opin. Plant Biol. 8:462-468.

Vanvolkenburgh, E., R.E. Cleland, and M. Watanabe. 1990. Light-stimulated cell expansion in bean (Phaseolus-Vulgaris L) leaves.2. Quantity and quality of light required. Planta 182:77-80.

Vince-Prue, D. and C.G. Guttridge. 1973. Floral initiation in strawberry: Spectral evidence for the regulation of flowering by long-day inhibition. Planta 110:165-172.

Vince-Prue, D., C.G. Guttridge, and M.W. Buck. 1976. Photocontrol of petiole elongation in lightgrown strawberry plants. Planta 131:109-114. 
Vinti, G., A. Hills, S. Campbell, J.R. Bowyer, N. Mochizuki, J. Chory, and E. Lopez-Juez. 2000. Interactions between hyl and gun mutants of Arabidopsis, and their implications for plastid/nuclear signalling. Plant J. 24:883894.

Wang, H., L.G. Ma, J.M. Li, H.Y. Zhao, and X.W. Deng. 2001. Direct interaction of Arabidopsis cryptochromes with COP1 in light control development. Science 294:154-158.

Weller, J.L., N. Beauchamp, L.H. Kerckhoffs, J.D. Platten, and J.B. Reid. 2001a. Interaction of phytochromes A and B in the control of deetiolation and flowering in pea. Plant J. 26: 283-294.
Weller, J.L., G. Perrotta, M.E. Schreuder, A. van Tuinen, M. Koornneef, G. Giuliano, and R.E. Kendrick. 2001b. Genetic dissection of blue-light sensing in tomato using mutants deficient in cryptochrome 1 and phytochromes A, B1 and B2. Plant J. 25:427-440.

Yanagi, T., T. Yachi, N. Okuda, and K. Okamoto. 2006. Light quality of continuous illuminating at night to induce floral initiation of Fragaria chiloensis L. CHI-24-1. Sci. Hort. 109:309-314.

Yorio, N., G. Goins, H. Kagie, R. Wheeler, and J. Sager. 2001. Improving spinach, radish, and lettuce growth under red light-emitting diodes (LEDs) with blue light supplementation. HortScience 36:380-383.
Zeugner, A., M. Byrdin, J.P. Bouly, N. Bakrim, B. Giovani, K. Brettel, and M. Ahmad. 2005. Light-induced electron transfer in Arabidopsis cryptochrome-1 correlates with in vivo function. J. Biol. Chem. 280:19437-19440.

Zhang, Y.C., S.F. Gong, Q.H. Li, Y. Sang, and H.Q. Yang. 2006. Functional and signaling mechanism analysis of rice CRYPTOCHROME 1. Plant J. 46:971-983.

Zhao, X., X. Yu, E. Foo, G.M. Symons, J. Lopez, K.T. Bendehakkalu, J. Xiang, J.L. Weller, X. Liu, and J.B. Reid. 2007. A study of gibberellin homeostasis and cryptochrome-mediated blue light inhibition of hypocotyl elongation. Plant Physiol. 145:106-118. 